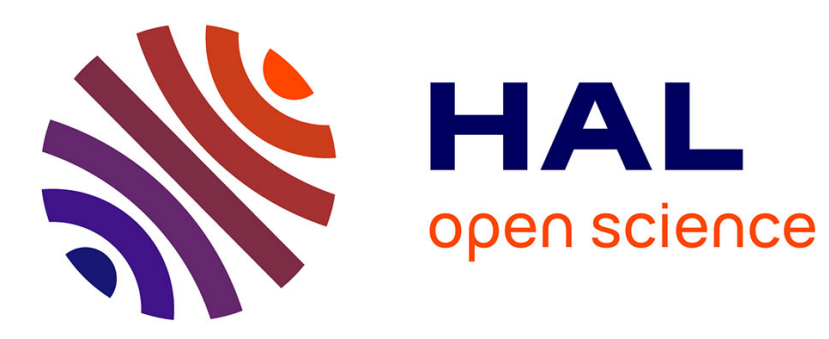

\title{
Cascades of energy and helicity in axisymmetric turbulence
}

Bo Qu, Aurore Naso, Wouter J.T. Bos

\section{To cite this version:}

Bo Qu, Aurore Naso, Wouter J.T. Bos. Cascades of energy and helicity in axisymmetric turbulence. Physical Review Fluids, 2018, 3 (1), pp.014607. 10.1103/PhysRevFluids.3.014607 . hal-01705327

\section{HAL Id: hal-01705327 \\ https://hal.science/hal-01705327}

Submitted on 9 Feb 2018

HAL is a multi-disciplinary open access archive for the deposit and dissemination of scientific research documents, whether they are published or not. The documents may come from teaching and research institutions in France or abroad, or from public or private research centers.
L'archive ouverte pluridisciplinaire HAL, est destinée au dépôt et à la diffusion de documents scientifiques de niveau recherche, publiés ou non, émanant des établissements d'enseignement et de recherche français ou étrangers, des laboratoires publics ou privés. 


\title{
Cascades of energy and helicity in axisymmetric turbulence
}

\author{
Bo Qu, Aurore Naso, and Wouter J. T. Bos \\ Laboratoire de Mécanique des Fluides et d'Acoustique, CNRS, École Centrale de Lyon, Université Claude \\ Bernard Lyon 1 and INSA de Lyon, 36 avenue Guy de Collongue, F-69134 Écully Cedex, France
}

(Received 21 July 2017; published 18 January 2018)

\begin{abstract}
A spectral analysis of strictly axisymmetric turbulence is performed. Both freely decaying and statistically steady flows are considered. In helical flows we identify a dual cascade, where energy is transferred towards the large scales and helicity to the smallest ones. It is shown that even in the absence of net helicity, a dual cascade persists, transferring energy backward and positively and negatively polarized helicity fluctuations forward.
\end{abstract}

DOI: 10.1103/PhysRevFluids.3.014607

\section{INTRODUCTION}

One of the key concepts in the study of turbulence is the existence of cascades of the inviscid global invariants of the Navier-Stokes equations. In three-dimensional (3D) turbulence, the two invariants are energy $E$ and helicity $H_{1}$. The direct cascade of energy represents the transfer of energy from large scales to smaller ones, at which dissipation becomes dominant [1]. In 3D homogeneous and isotropic turbulence, such a scenario is compatible with an energy spectrum $E(k)$ scaling proportional to $k^{-5 / 3}$ ( $k$ being the wave number) in the inertial range of scales [2], a result remarkably well verified in a wide range of high Reynolds number flows. The cascade of helicity was first discussed by Brissaud et al. [3], who concluded that two scenarios were possible: in the first one, the forward cascades of energy and helicity would coexist, whereas in the second one a forward cascade of helicity would be accompanied by an inverse cascade of energy. The latter possibility, where a "pure" helicity cascade is expected in a range of scales in which the energy cascade is inhibited by that of helicity, gives rise to a helicity spectrum of the form $H_{1}(k) \sim k^{-4 / 3}$, which would lead in the same range of scales to an energy spectrum of the form $E(k) \sim k^{-7 / 3}$. However, such a scaling is not observed in 3D isotropic turbulence, most probably due to the fact that helicity is not a definite positive quantity like energy [4]. Numerical simulations [5] rather support the first scenario, according to which energy and helicity cascade simultaneously forward, with a scaling behavior $H_{1}(k) \sim k^{-5 / 3}$ and $E(k) \sim k^{-5 / 3}$.

The shape of the energy spectra for two-dimensional (2D) turbulence was predicted by Kraichnan [6,7] and Batchelor [8]. Kraichnan predicted in particular the existence of a dual cascade [6] in the case where an isotropic 2D turbulent flow is sustained by an external forcing acting at a specific wave number $k_{f}$. In this case, the kinetic energy is expected to cascade towards large scales (small wave numbers, $k<k_{f}$ ) and the enstrophy towards small scales (large wave numbers, $k>k_{f}$ ). Dimensional analysis, using the assumption of local energy transfer, then leads to an energy spectrum of the form $E(k) \sim k^{-5 / 3}$ in the inverse cascade regime $\left(k<k_{f}\right)$, and $E(k) \sim k^{-3}$ in the direct cascade one $\left(k>k_{f}\right)$, with possible logarithmic corrections [7]. This prediction of the existence of a dual cascade has been corroborated by high-resolution direct numerical simulations [9].

The nature of the cascades of invariants differs thus drastically between 2D and 3D turbulence. We focus here on turbulent cascades in strictly axisymmetric turbulence, a case intermediate between 2D and 3D turbulence, and for which almost no results are currently available, our recent investigation [10] being one of the rare exceptions. However, a spectral analysis of the von Kármán flow, whose time average is axisymmetric, was recently performed [11] and the existence of a dual cascade was evidenced in this system. In that study, spatial power spectra of the velocity fluctuations were 
measured. As the mean flow was observed to be Beltrami (i.e., velocity and vorticity are aligned), the helicity had a well-defined sign. For such flows the equality $H_{1}(k)=k E(k)$ holds. This might lead to the blocking of the energy cascade (the alternative scenario proposed for 3D turbulence in Ref. [3]). Based on this assumption, the authors hypothesized that helicity dominates the direct cascade (largest $k$ ), and that energy dominates the inverse one (towards small $k$ ) [11]. This scenario is compatible with the slopes of the experimentally measured energy spectra, that is, the inverse cascade is characterized by $E(k) \sim k^{-1}$ (a scaling compatible with a nonlocal energy cascade), and the direct cascade by a power law with an exponent between -2 and $-7 / 3$ (compatible with a local or nonlocal helicity cascade), depending on the Reynolds number. The terms local and nonlocal indicate here whether the transfer is local in wave number space as in a stepwise cascade process or dominated by nonlocal interactions involving the forcing scale (see, for instance, Ref. [12]). In the local case, the cascades are described by the classical Kolmogorov picture, whereas in the nonlocal case the time scale in the cascade is given by a large-scale shearing time, associated with a mean velocity gradient. In these experiments, only the time-averaged flow was axisymmetric and of Beltrami type. We consider here a more idealized situation in which the instantaneous flow is axisymmetric.

We will therefore focus here on strictly (that is, instantaneously) axisymmetric turbulence. This is an idealized situation, very difficult to approach in real flows except perhaps in confined nuclear fusion devices such as tokamaks [13]. However, there has been in the last decade a growing interest in this system, since the tools based on statistical mechanics and developed for 2D flows can be transposed to it [14-17], and because this type of theoretical analysis was found to give some results in qualitative agreement with those obtained for the mean (real) von Kármán flow [18-20].

More specifically, we perform in the present paper a spectral analysis of axisymmetric turbulence. For this, we will investigate possible cascades of the first quadratic invariants of the axisymmetric Euler equations, i.e., energy $E^{\text {tot }}$ and helicity $H_{1}^{\text {tot }}$ (the total quantities will be denoted hereafter with a superscript $t o t$, to distinguish them from their spectral density counterparts). In Ref. [10] it was in particular shown that in freely decaying axisymmetric turbulence helicity decays much faster than energy, a phenomenon still unexplained. This may be due to the different natures of the helicity and energy cascades. In particular, if the presence of a direct cascade of helicity and of an inverse cascade of energy is evidenced in this system, this will imply that helicity may have a tendency to be dissipated faster than energy, due to viscous effects.

We will test these hypotheses by performing a spectral analysis of decaying and of forced axisymmetric turbulence. The flows will be calculated by direct numerical simulation. The numerical methodology will be first described in Sec. II. A spectral analysis of axisymmetric turbulence in both the decaying and the forced cases will then be performed in Sec. III. Section IV concludes the paper.

\section{METHODOLOGY}

\section{A. Invariants}

The system we consider is described by the Navier-Stokes equations in cylindrical coordinates $(r, \theta, z)$, in which all the azimuthal derivatives $\partial_{\theta}$ are set to zero:

$$
\begin{gathered}
\partial_{t} u_{r}+u_{r} \partial_{r} u_{r}+u_{z} \partial_{z} u_{r}=-\partial_{r} p / \rho+\mathcal{V}_{r}+F_{r}+r^{-1} u_{\theta}^{2}, \\
\partial_{t} u_{\theta}+r^{-1} u_{r} \partial_{r}\left(r u_{\theta}\right)+u_{z} \partial_{z} u_{\theta}=\mathcal{V}_{\theta}+F_{\theta}, \\
\partial_{t} u_{z}+u_{r} \partial_{r} u_{z}+u_{z} \partial_{z} u_{z}=-\partial_{z} p / \rho+\mathcal{V}_{z}+F_{z}, \\
r^{-1} \partial_{r}\left(r u_{r}\right)+\partial_{z} u_{z}=0,
\end{gathered}
$$


where $\boldsymbol{u}$ and $p$ are the velocity and pressure fields, respectively, $\rho$ is the fluid density, and the $\mathcal{V}$ and $F$ terms represent the viscous and forcing contributions, respectively. In particular,

$$
\begin{aligned}
& \mathcal{V}_{r}=v\left[r^{-1} \partial_{r}\left(r \partial_{r} u_{r}\right)+\partial_{z z} u_{r}-r^{-2} u_{r}\right], \\
& \mathcal{V}_{\theta}=v\left[r^{-1} \partial_{r}\left(r \partial_{r} u_{\theta}\right)+\partial_{z z} u_{\theta}-r^{-2} u_{\theta}\right], \\
& \mathcal{V}_{z}=v\left[r^{-1} \partial_{r}\left(r \partial_{r} u_{z}\right)+\partial_{z z} u_{z}\right],
\end{aligned}
$$

where $v$ is the kinematic viscosity of the fluid.

This axisymmetric system bears similarities to both 2D and 3D flows. Indeed, it describes the dynamics of three velocity components (as in three dimensions), but these components vary only in two directions (as in two dimensions), $r$ and $z$. The last term in the evolution equation for $u_{r}$, Eq. (1), however, shows the difference with Cartesian two-dimensional, three-component (2D3C) flows [21], in which one of the velocity components is passively advected by the other two. Due to the presence of this term in the present setting, the quantity $u_{\theta}$ is not passively advected by the $\left(u_{r}, u_{z}\right)$ flow, and the dynamics of all three velocity components are interdependent.

In the absence of forcing and viscosity, this system conserves several quantities, in the following called the invariants of the system [14]. The quantities that we will focus on are the quadratic invariants, i.e., kinetic energy,

$$
E^{\mathrm{tot}}=\frac{1}{2}\left\langle u_{r}^{2}+u_{\theta}^{2}+u_{z}^{2}\right\rangle
$$

and helicity

$$
H_{1}^{\mathrm{tot}}=2\left\langle u_{\theta} \omega_{\theta}\right\rangle
$$

where $\omega_{\theta}=\boldsymbol{e}_{\theta} \cdot(\nabla \times \boldsymbol{u})$ is the azimuthal component of vorticity $\boldsymbol{\omega}$ and $\langle\cdots\rangle$ is the integral over the fluid domain (in axisymmetric flows, $\left\langle u_{\theta} \omega_{\theta}\right\rangle=\left\langle u_{r} \omega_{r}+u_{z} \omega_{z}\right\rangle$ ). Apart from energy and helicity, axisymmetric turbulence conserves an infinite number of quantities in the absence of viscosity [14]. These quantities are the moments of the angular momentum

$$
I_{m}^{\mathrm{tot}}=\left\langle\left(r u_{\theta}\right)^{m}\right\rangle
$$

and the generalized helicities

$$
H_{n}^{\mathrm{tot}}=\left\langle r^{-1}\left(r u_{\theta}\right)^{n} \omega_{\theta}\right\rangle,
$$

for $m \geqslant 1$ and $n \geqslant 0$. We have chosen to focus on the quadratic quantities, $E^{\text {tot }}$ and $H_{1}^{\text {tot }}$, because of the analogy with 2D and with 3D turbulence, and because in a previous study [11] experimental energy spectra measured in (statistically axisymmetric) turbulence were interpreted in terms of these quantities. The nonquadratic quantities (circulation $H_{0}$ and higher order moments, $I_{m}^{\text {tot }}$ for $m \geqslant 1$ and $H_{n}^{\text {tot }}$ for $n \geqslant 2$ ) will not be considered in the present investigation since their influence on the cascade directions is supposed to be small, as is the case for the high-order moments of vorticity in 2D turbulence.

\section{B. Description of the spectral method}

To integrate the axisymmetric Navier-Stokes equations in a cylindrical domain of radius $R$ and height $L$, we used a fully spectral method based on an expansion of the velocity field in a basis consisting of Chandrasekhar-Kendall (CK) eigenfunctions of the curl $\boldsymbol{A}_{n q}$, defined such that

$$
\nabla \times \boldsymbol{A}_{n q}=\lambda_{n q} \boldsymbol{A}_{n q} .
$$

An existing code solving the Navier-Stokes equations in a circular geometry [22] was modified and further developed to this aim. For these basis functions no fast transform (such as the fast Fourier transform) exists, and the attainable resolution is limited by the fact that the cost of the evaluation of the convolution products is a rapidly increasing function of the resolution. Therefore the results in this work will be accurate but limited to Reynolds numbers at which the inertial ranges are not very 
extensive. Assuming periodic boundary conditions in the axial direction and nonpenetration in the radial one, $\boldsymbol{A}_{n q}$ is a combination of Fourier modes in the $z$ direction and of Bessel functions in the radial one. $n$ and $q$ are the mode numbers, respectively, associated to the axial and radial directions: $k_{n}$ is the axial wave number, and $\gamma_{q}$ is the $q$ th zero of the Bessel function $J_{1}$ in the radial direction. These latter quantities are related to $\lambda_{n q}$ by the relation

$$
\lambda_{n q}^{2}=k_{n}^{2}+\gamma_{q}^{2} \equiv k(n, q)^{2},
$$

and $\lambda_{n q}$ can have both positive and negative values, indicated by $\lambda_{n q}^{+}$and $\lambda_{n q}^{-}$, respectively. Relation (11) defines thus an effective wave number $k(n, q)$ as the norm of $\lambda_{n q}$. In the following this wave number will be indicated $k$ omitting its dependence on $n, q$ in the notation.

The modes $A_{n q}$ form an orthonormal basis of solenoidal functions [23] (see also Refs. [15,24]), allowing a precise, nondiffusive representation of the dynamics of the velocity field, represented by the coefficients $\xi_{n q}(t)$,

$$
\boldsymbol{u}(r, z, t)=\sum_{n q} \xi_{n q}(t) \boldsymbol{A}_{n q}(r, z)
$$

The discretization of the Navier-Stokes equations in the spectral space of CK modes then reads

$$
\begin{aligned}
\frac{\partial}{\partial t} \xi_{n q}= & \sum_{n^{\prime} q^{\prime}} \sum_{n^{\prime \prime} q^{\prime \prime}} \lambda_{n^{\prime \prime} q^{\prime \prime}} \xi_{n^{\prime} q^{\prime}} \xi_{n^{\prime \prime} q^{\prime \prime}} \frac{1}{V} \int_{r, \theta, z} A_{n q}^{*} \cdot\left(A_{n^{\prime} q^{\prime}} \times A_{n^{\prime \prime} q^{\prime \prime}}\right) r d r d z d \theta \\
& -v \lambda_{n q}^{2} \xi_{n q}+\alpha(n, q) \nu \lambda_{n q}^{2} \xi_{n q},
\end{aligned}
$$

where the last term is a negative viscosity forcing term commonly used in 3D isotropic turbulence [25], set to zero in simulations of decaying turbulence. The function $\alpha(n, q)$ measures its amplitude and is nonzero only in a narrow band of wave numbers (its value is then larger than 1). One of the advantages of the forcing scheme we use is that it conveniently permits to consider two different configurations of statistically stationary flows: helical ones, with a finite (and therefore of well-defined sign) global helicity, and nonhelical ones (zero global helicity). One or the other regime can be imposed easily by tuning the forcing term. Details on the procedure can be found in Ref. [26].

In the case of two-dimensional turbulence in a periodic domain, the energy cumulates at large scales if no additional large-scale dissipation is added. In forced simulations a friction term is therefore often added to the system in order to allow the system to obtain a statistically steady state. When solid boundaries are present, enstrophy generation at the walls constitutes an efficient energy dissipation mechanism and no further friction is needed [27]. This will also be observed in the present simulations, where steady states are attained without other damping than the viscous dissipation term.

Equation (13) is integrated using a fourth order Runge-Kutta time integration scheme. The time step is calculated instantaneously by setting the dimensionless Courant number based on the rootmean square of the velocity fluctuations to 0.05 , a value found to be optimal in our numerical setup. Simulations are carried out in a rectangular domain representing an $(r, z)$ plane of a cylinder of radius $R$ and height $L$. Further details on the method are provided in Refs. [10,26].

\section{Spectral decomposition of energy and helicity}

The total kinetic energy can be written as

$$
E^{\mathrm{tot}}=\frac{1}{2} \sum_{n=0, q}^{ \pm \lambda} \xi_{n q}^{*} \xi_{n q}+\sum_{n>0, q}^{ \pm \lambda} \xi_{n q}^{*} \xi_{n q} .
$$

The notation $\sum^{ \pm \lambda}$ means that the summation is performed over the modes of positive and negative $\lambda$. The energy spectrum $E(k)$ is calculated by considering the suitable modes of Eq. (14), where the link between $(n, q)$ and $k$ is given by relation $(11)$. In practice, when the spectra are evaluated 
numerically, we will define concentrical shells in the $(n, q)$ plane around discrete values of $k$, as is common practice in spectral calculations. Similarly we define the total helicity,

$$
H_{1}^{\mathrm{tot}}=\sum_{n=0, q}^{ \pm \lambda} \xi_{n q}^{*} \xi_{n q} \lambda_{n q}+\sum_{n>0, q}^{ \pm \lambda} 2 \xi_{n q} \xi_{n q} \lambda_{n q},
$$

and the helicity spectrum is defined analogously to $E(k)$. The cascades of energy and helicity are related to the fluxes of these quantities between length scales. To determine these fluxes, we first introduce the spectral balance equations for energy and helicity, which can be derived from the modally decomposed form of the Navier-Stokes equations and formally read

$$
\partial_{t} E(k, t)=T_{E}(k, t)-2 \nu k^{2} E(k, t)+F_{E}(k, t)
$$

and

$$
\partial_{t} H_{1}(k, t)=T_{H_{1}}(k, t)-2 \nu k^{2} H_{1}(k, t)+F_{H_{1}}(k, t),
$$

where $T_{E}$ and $T_{H_{1}}$ are the transfer functions, and $F_{E}$ and $F_{H_{1}}$ are the input spectra of energy and helicity by the forcing. Relation (11) is used here to express the dynamics as a function of $k$ only. Since energy and helicity are both inviscid invariants of the unforced axisymmetric Navier-Stokes equations, the transfer functions satisfy the integral relations:

$$
\int_{0}^{\infty} d k T_{X}(k, t)=0
$$

where $X=E, H_{1}$, respectively, denotes energy and helicity. By definition, the transfer rate or flux of an inviscid invariant $X$ through the wave number $k$ is then

$$
\Pi_{X}(k, t)=-\int_{0}^{k} T_{X}\left(k^{\prime}, t\right) d k^{\prime}
$$

and quantifies the possible cascade of the invariant $X$ through scale space.

\section{RESULTS}

Two typical types of flows will be considered hereafter: decaying flows starting from well-defined initial conditions and statistically steady flows.

\section{A. Decaying turbulence}

We start by carrying out a spectral analysis in the decaying case, which will guarantee that the results obtained are not spurious effects of the forcing scheme and will allow us to interpret straightforwardly the results obtained in Ref. [10] in the same numerical setup. The results presented in this subsection were obtained in a cylindrical domain of radius and height $R=L=2 \pi$. The numbers of modes considered in the axial and radial directions were $n_{\max }=40$ and $q_{\max }=60$, respectively, a resolution checked to be sufficient for the flows investigated [26]. The kinematic viscosity was set to $v=10^{-2}$, and the total initial energy in the flow $E^{\text {tot }}$ was fixed so that the initial large-scale Reynolds number, defined as $\operatorname{Re}=U L / v$ with $U=\sqrt{2 E^{\text {tot }} / 3}$, is around 643. Other calculations, conducted with different values of the domain aspect ratio $R / L$ and of viscosity, led to the same results. In the following, time will be normalized by the initial eddy turnover time defined as $\tau=\ell(t=0) / U(t=0)$, where the integral scale is defined as

$$
\ell=\frac{\int k^{-1} E(k) d k}{\int E(k) d k} .
$$

We will first try to evidence the existence of a direct cascade. For this, initial conditions in which the modal energy is concentrated at the smallest wave numbers are used. A modal representation of 


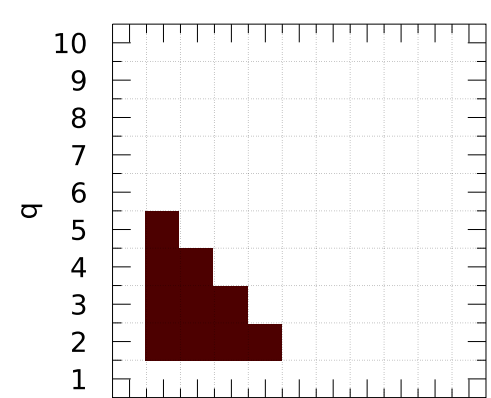

$0 \begin{array}{lllllllll}0 & 2 & 4 & 5 & 6 & 7 & 8 & 9 & 10\end{array}$

(a)

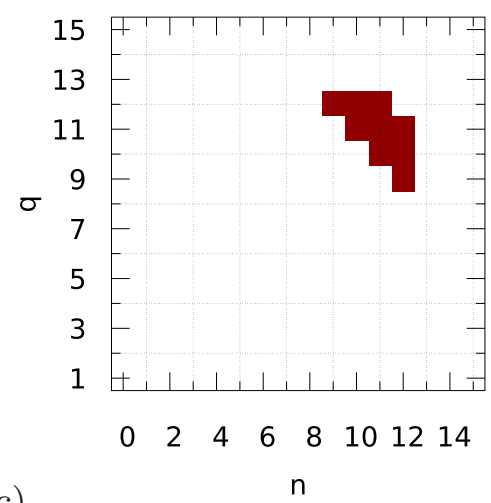

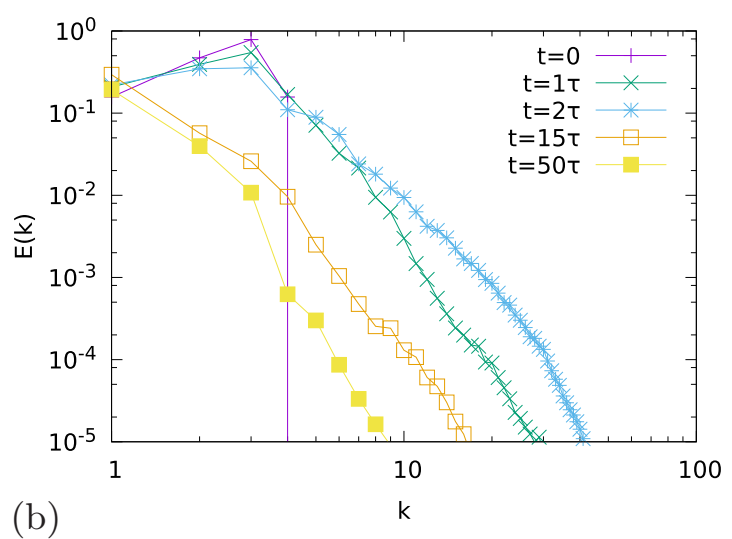

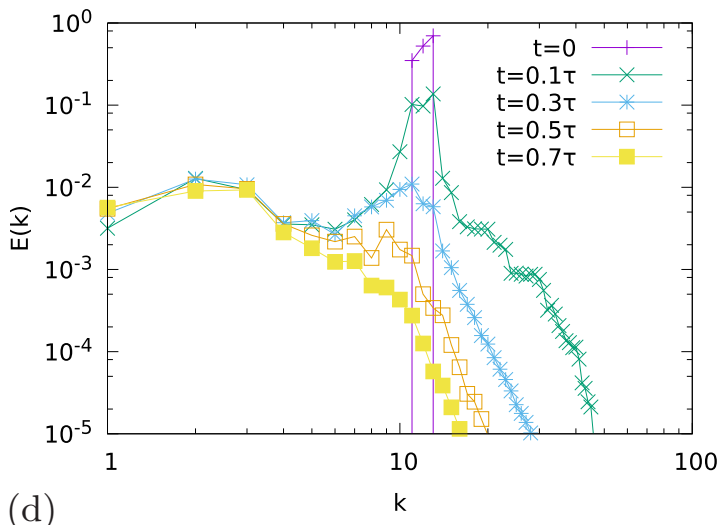

FIG. 1. Decaying turbulence. Left column: modal representation of the initial condition; energy is concentrated at (a) small wave numbers and (c) intermediate wave numbers. Right column: energy spectra at different times (each figure has been obtained using the initial condition represented on its left); in (b), a direct cascade is visible, in (d) both direct and inverse cascades seem to exist.

the initial condition is shown in Fig. 1(a). The time evolution of the energy spectrum for this case is displayed in Fig. 1(b). The existence of a direct cascade, from the small to the large wave numbers, is clearly visible. Inertial ranges, that would be characterized by the existence of power law behaviors of the spectrum, cannot be evidenced due to the low values of the Reynolds numbers obtained and the inherent spectral imbalance of decaying turbulence. We will attempt to evidence the presence of inertial ranges in the following by considering statistically steady turbulence.

In order to see if there also exist inverse cascades, we conducted other calculations in which the initial modal energies are concentrated at intermediate values of the wave number [Fig. 1(c)]. The spectra displayed in Fig. 1(d) show that both direct and inverse cascades seem to occur.

Some clear evidence for the existence of a dual cascade has been illustrated here for two typical initial conditions, but the same results hold in all the flows that we have considered (helical or nonhelical flows, vanishing or finite angular momentum, etc.). We have also considered initial conditions in which the inverse cascade is permitted only for the radial or for the axial wave number by considering initial conditions where the energy was centered around $(n, q)=(0, q)$ and $(n, 1)$ respectively, and found a similar dual cascade behavior. The present analysis supports a dual cascade scenario but gives no information about the quantities associated to these cascades. To assess this, a detailed flux balance is needed and will be performed in the following sections. 

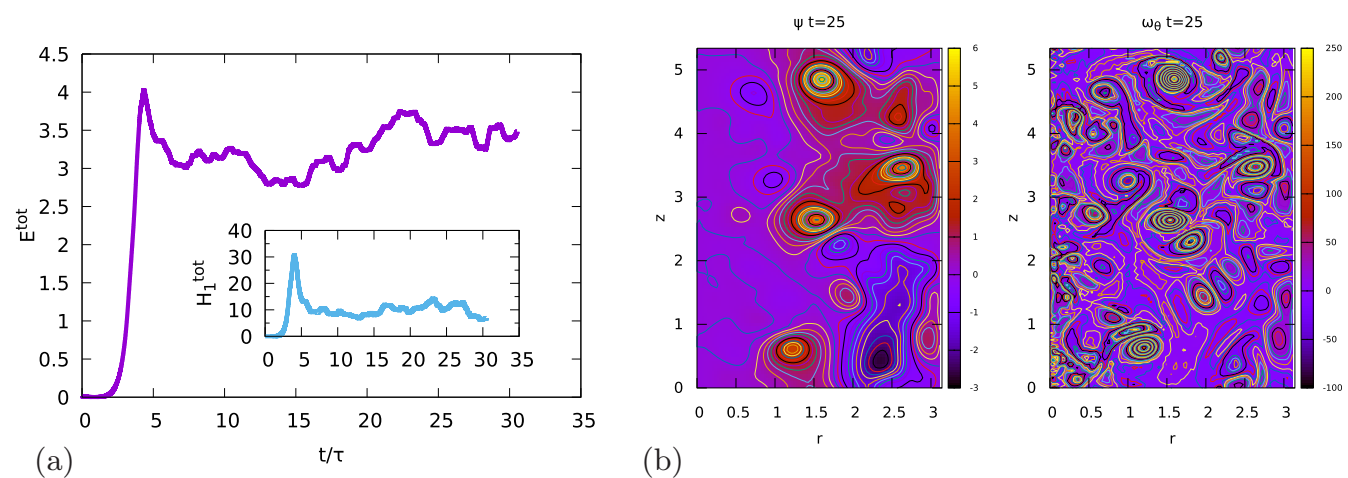

FIG. 2. Forced helical flow: (a) time evolution of the total energy and helicity; (b) typical flow visualizations at steady state. The quantities $\psi$ and $\omega_{\theta}$ are, respectively, the stream function and the azimuthal vorticity component.

\section{B. Steady helical flows}

We now turn to the investigation of statistically stationary flows. These flows were computed in a cylindrical domain of radius $R=\pi$ and height $L=1.7 \pi$ (similar results were obtained in a domain in which $R=L$ ). The maximal numbers of modes in the axial and radial directions were $n_{\max }=45$ and $q_{\max }=52$, and the viscosity was set to $v=5 \cdot 10^{-3}$. The forcing term was nonzero in a narrow band of intermediate wave numbers: $k \in[12,15]$, and its magnitude was $\alpha=3$.

As illustrated in Fig. 2(a), the helically forced flow reaches a statistically stationary state for $t / \tau \gtrsim 5$. As expected, in this regime the total helicity fluctuates around a nonzero value. As previously explained, no large-scale friction was needed to prevent the accumulation of energy at large scale, despite the inverse energy cascade occurring in the system, very likely because of the natural viscous damping at the cylindrical wall. Indeed, it is known that in 2D turbulence in the absence of walls an additional friction is needed to dissipate the energy which otherwise accumulates at large scales [6]. The interaction of a large scale with a wall provides an alternative. When a very large scale interacts with a wall, a long shear layer will indeed form along it. In the wall-normal direction, such shear layers will become very thin, thereby contributing to the high wave numbers at which the energy dissipation is non-negligible. The energy dissipation mechanism due to the presence of walls in 2D turbulence is thereby probably associated with these very anisotropic structures. The fact that no additional friction is required to prevent the energy from accumulating at large scales may also be explained by alternative mechanisms. Scenarios according to which simultaneous upward and downward cascades coexist were in particular recently evidenced in 2D3C flows [28] but do not need to be invoked here to explain the damping because of the presence of walls.

Figure 2(b) shows typical instantaneous flow fields in the stationary state. The characteristic scales of the structures are consistent with the fact that most of the energy is concentrated at intermediate wave numbers, a property that will be also clear in the corresponding energy spectra. This situation is different from the decaying case, in which energy eventually concentrates in the smallest wave numbers and the final flow therefore exhibits large-scale structures [10].

The energy spectra associated with this flow are shown at different times in Fig. 3(a). The initial conditions are such that energy is concentrated at wave numbers close to the forcing scale. A dual cascade clearly develops, as already observed in our simulations of decaying turbulence in Sec. III A. In the stationary regime, the spectra display a scaling which seems compatible with $E(k) \sim k^{-5 / 3}$ for the inverse cascade and $E(k) \sim k^{-6}$ for the direct one.

Based on dimensional grounds, the $E(k) \sim k^{-5 / 3}$ scaling measured at small wave numbers is compatible with the existence of an energy cascade. As already mentioned in the introduction, energy is also transferred from the small to the large scales in 2D turbulence, due to the blocking of its direct cascade by the enstrophy, which cascades itself from the large to the small scales. In 

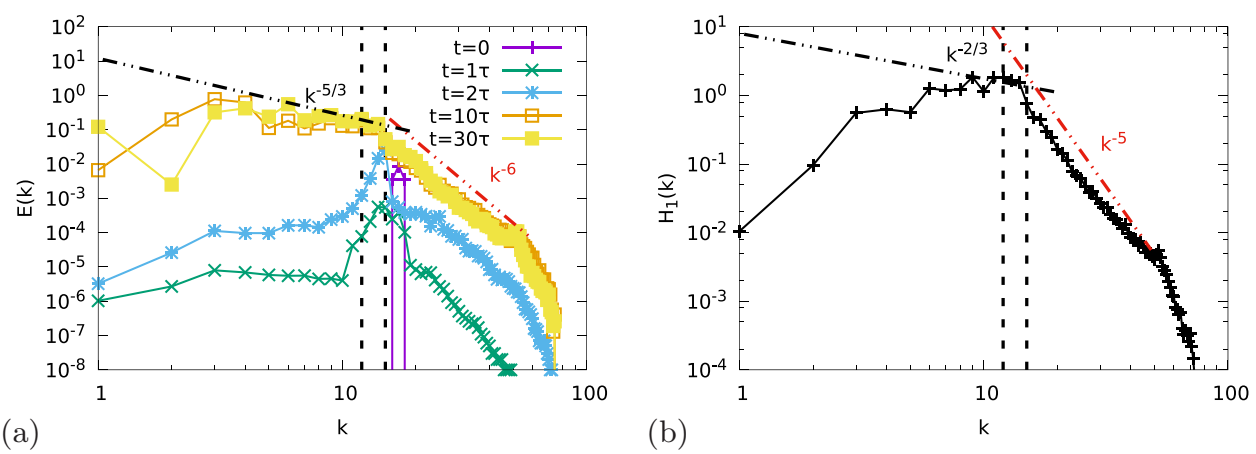

FIG. 3. Wave number spectra in the forced helical case: (a) energy spectra at different times; (b) helicity spectrum time averaged over the interval $t / \tau \in[10,30]$. The vertical dashed lines indicate the wave number range in which the forcing is applied.

axisymmetric turbulence, a plausible assumption is that the energy cascade might be inhibited by that of helicity.

More precisely, the helicity and energy spectra satisfy, by definition, the relation $H_{1}(k) \leqslant k E(k)$, formally analogical to the relation $\Omega(k)=k^{2} E(k)$ commonly used in 2D turbulence, where $\Omega(k)$ is the enstrophy spectrum. When helicity is maximal (as, e.g., is the case in Beltrami flows), this relation turns into an equality: $H_{1}(k)=k E(k)$ [11]. Such a relation seems to be satisfied in the present setup [compare Figs. 3(a) and 3(b)]. The helicity flux, if it exists, is therefore expected to be dominant with respect to the energy one at large wave numbers, while the energy flux should dominate at small $k$. In other words, a direct helicity cascade and an inverse energy cascade are expected to occur in this system. The helicity cascade to the smallest scales would thus block the direct energy cascade, and $E$ would be rather transferred to the largest scales. Such a scenario is compatible with those already proposed for the von Kármán experiment [11] or (unsuccessfully) for homogeneous and isotropic turbulence [3]. It will be tested hereinafter in axisymmetric flows by measuring the transfer rates of energy and helicity.

On a side note, a forward helicity cascade would lead dimensionally to a scaling $E(k) \sim k^{-7 / 3}$ $[3,11]$, which is not compatible with the very steep slope, $E(k) \sim k^{-6}$, measured in the large wave numbers range (Fig. 3). However, such dimensional predictions assume asymptotically large Reynolds numbers and locality of scale interactions. These assumptions are not necessarily satisfied here. In particular, at the scales $k>k_{f}$ the effect of viscous dissipation can certainly not be neglected.

In Fig. 3(b) is shown the time-averaged helicity spectrum. Direct and inverse cascades are clearly visible for this quantity as well. As already mentioned, these spectra seem to satisfy the relation $H_{1}(k) \sim k E(k)$, that is, $H_{1}(k) \sim k^{-2 / 3}$ at small wave numbers and $H_{1}(k) \sim k^{-5}$ at large $k$.

An advantage of numerical simulations is that they allow us to measure relatively straightforwardly the fluxes of inviscid invariants, so as to verify the physical assumptions behind the cascade scenarios. The time averages of $\Pi_{E}$ and $\Pi_{H_{1}}$ [defined by relation (19) averaged during the statistically steady state over the interval $t / \tau=[10,30])$ are plotted in Fig. 4. Two directions of transfer are clearly visible: on average, the fluxes are negative at wave numbers smaller than $k_{f}$, the forcing wave number, and positive for $k>k_{f}$. The Reynolds number of the flow is too low for getting plateaus, which would indicate inertial ranges. Nevertheless, Fig. 4(a) shows unambiguously that the inverse energy flux is more dominant than its direct counterpart, a signature of an inverse energy cascade, while the opposite behavior occurs in Fig. 4(b), reflecting the existence of a direct helicity cascade. The scenario proposed above is therefore confirmed, even though no asymptotic scaling is obtained, as expected at the moderate Reynolds numbers considered. This remark holds in particular for the direct cascade, for which the observed spectra are approximately proportional to $k^{-6}$. In this range of scales, the viscous dissipation is far from negligible so that no further interpretations of this range as an inertial one will be attempted here. 


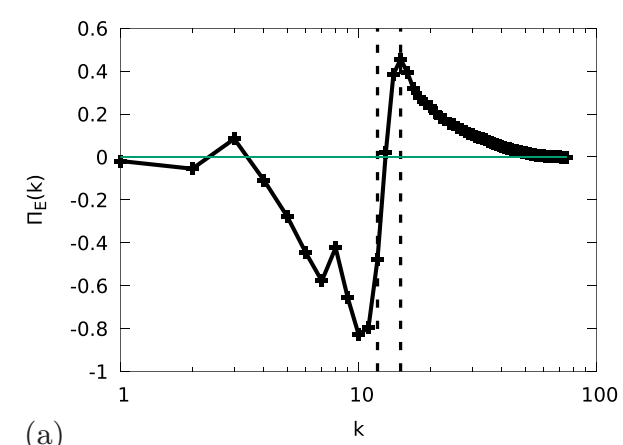

(a)

FIG. 4. Flux of (a) energy and (b) helicity, time averaged over the interval $t / \tau \in[10,30]$, for the forced helical flow. The vertical dashed lines indicate the wave numbers range in which the forcing is applied.

It has been known for a long time that in the absence of viscosity the dynamics of a finite number of modes will yield equilibrium distributions in which the different modes are in thermal equilibrium [29]. The directions of the fluxes $\Pi_{X}$ are then in general determined by the inviscid equilibrium solutions of the system [6], and the system will try to relax towards these states. In the presence of viscous damping, such a state is in general not attained, but for the inviscid state, considering a finite number of modes, the flux will vanish when the equilibrium state is reached and the energy will be statistically equidistributed over the different modes. The definition of the energy spectrum will then, in the present case, yield a spectrum proportional to $k$. In the presence of helicity, the equilibrium spectrum $H_{1}(k)$ for 3D turbulence was discussed by Kraichnan [30] and expected to scale as $H_{1}(k) \sim k^{2}$. In the absence of viscosity, we have checked the relaxation of our system to inviscid equilibrium and have observed the final state in which $E(k) \sim k$ and $H_{1}(k) \sim k^{2}$. Note that these distributions are very different from the $k^{-5 / 3}$ inverse energy cascade and $k^{-7 / 3}$ direct helicity cascade ranges discussed in the introduction. As a consequence, this should not constitute a problem for distinguishing between equilibrium and constant flux states.

\section{Steady helicity-free flow}

As stated before, our forcing allows us to consider both helical and helicity-free flows. In this section we remove the average helicity injection from the forcing scheme and otherwise keep the same numerical setup, including parameter values, as in Sec. III B. The resulting flow reaches a statistically steady state with a kinetic energy fluctuating around a mean value, as illustrated in Fig. 5(a). In this
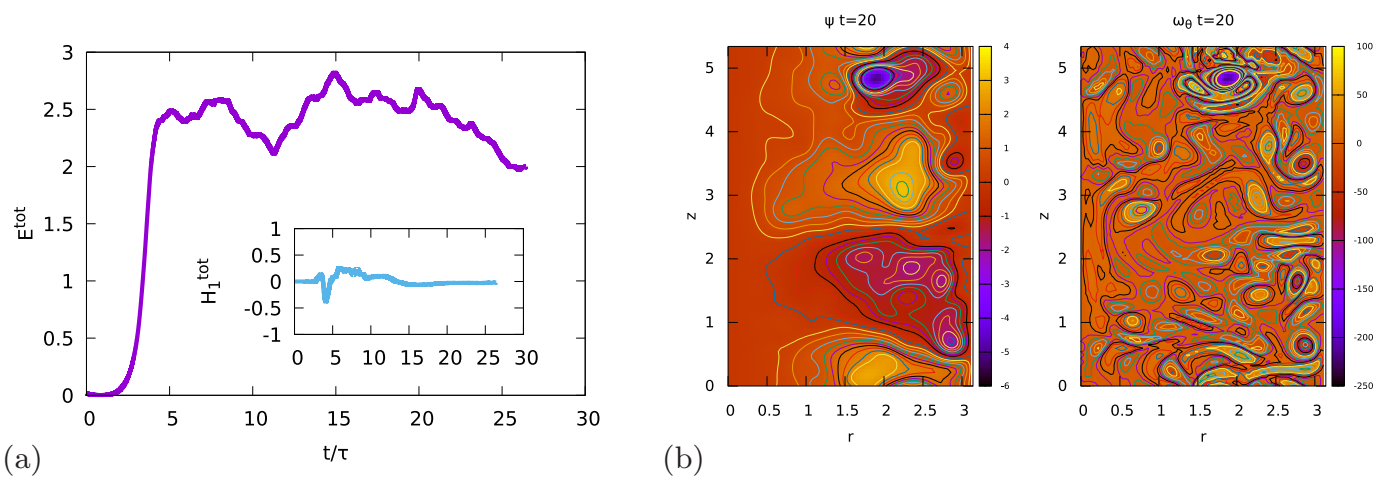

(b)

FIG. 5. Forced nonhelical flow: (a) time evolution of the total energy and helicity; (b) flow visualizations. The same conventions as in Fig. 2. 

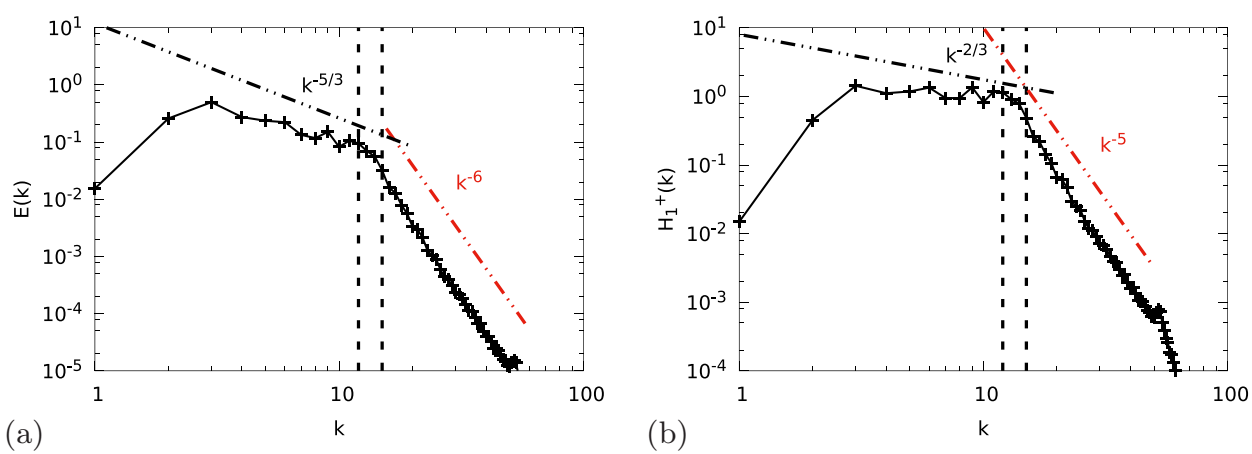

FIG. 6. Spectra for the forced, nonhelical flow time-averaged over the interval $t / \tau \in[16,26]$ : (a) energy spectrum; (b) spectrum of helicity of the positively polarized component (the spectrum for the negatively polarized component, not shown here, displays the same behavior). The same conventions as in Fig. 3.

regime, the total helicity fluctuates around zero. Typical flow visualizations are shown in Fig. 5(b), which can be compared to those obtained in the helical case [Fig. 2(b)]. No significant qualitative difference between these fields is visible.

The energy spectrum is shown in Fig. 6(a). A clear similarity is observed with the one obtained in the helical case. This observation is puzzling. Indeed, if it is the helicity which blocks the energy flux towards the small scales, as was suggested in the previous section, the spectral energy distribution might be expected to drastically change in its absence. This seems not to be the case: a scaling suggestive of an inverse cascade is present also in this flow.

We will attempt to give here a possible explanation of this observation. The presence of a net global helicity does not seem to drastically change the nature of the energy cascade. However, helicity can always be finite locally, and an arbitrary velocity field can be written as the sum of helical modes [31], a decomposition used for instance to study the role of helicity using shell models [32-34]. Recent studies have considered Navier-Stokes turbulence where artificially only helical modes of a single polarity were retained [35,36]. In these flows, an inverse cascade occurs, even in the 3D isotropic case. We will use the same concepts to study the present flow. Our numerical method allows us to write straightforwardly the total helicity as the sum of two components of opposite polarities, a decomposition of the flow formally very similar to the helical wave decomposition $[4,31,34,37]$ for incompressible velocity fields.

Using our discretization, the positive or negative polarity contributions to the flow are directly associated to the eigenvalues $\lambda_{n q}$ [whose absolute value is given in Eq. (11)] of the same sign. More precisely, we can consider the contributions of the positively (or negatively) polarized modes only, by replacing the sums in expressions (14) and (15) over all modes by a sum exclusively over the modes of $\lambda_{n q}$ of the same sign. The positively polarized energy, for instance, reads

$$
E^{+, \text {tot }}=\frac{1}{2} \sum_{n=0, q}^{+\lambda} \xi_{n q}^{*} \xi_{n q}+\sum_{n>0, q}^{+\lambda} \xi_{n q}^{*} \xi_{n q},
$$

where the notation $\sum^{+\lambda}$ means that the summation is performed over the modes of positive $\lambda$ only. This expression allows us to define also the spectrum $E^{+}(k)$ by summing the terms $E^{+}(n, q)$ in concentrical shells of the $(n, q)$ plane around discrete values of $k . E^{- \text {,tot }}$ and $E^{-}(k)$ are defined in the same way, and the expressions of $H_{1}^{ \pm \text {,tot }}$ and $H_{1}^{ \pm}(k)$ are obtained analogously from expression (15).

We have plotted in Fig. 6(b) the resulting spectrum $H_{1}^{+}(k)$ in the nonhelical case [the spectrum $H_{1}^{-}(k)$, not shown, is identical]. Comparing this figure with Fig. 3(b) shows that $H_{1}^{ \pm}(k)$ satisfy the same scaling as the total helicity spectrum in the helical flow (incidentally, the same result holds for 


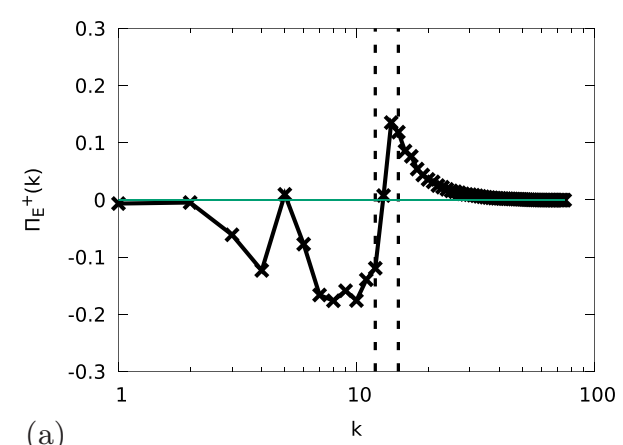

(a)

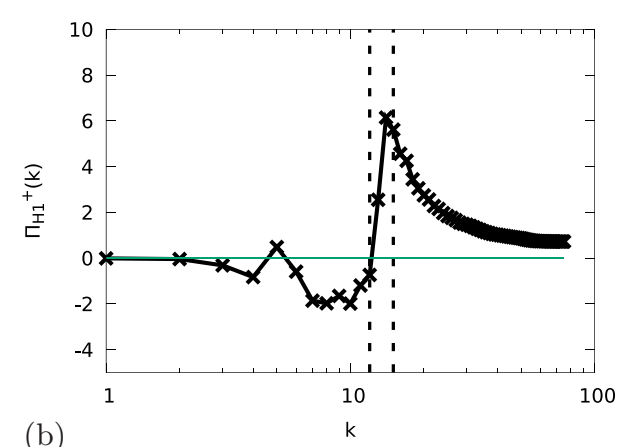

(b)

FIG. 7. Flux of (a) positively polarized energy and (b) positively polarized helicity time-averaged over the interval $t / \tau \in[16,26]$, for the forced nonhelical flow. The negatively polarized quantities and the total energy flux, not shown here, display similar behaviors. The same conventions as in Fig. 4.

the helical flow). The spectra of energy for the two polarities, $E^{+}(k)$ and $E^{-}(k)$, have been found to scale like $E(k)$ in both the helical and nonhelical cases as well.

Since the quantities $E^{ \pm \text {,tot }}$ and $H_{1}^{ \pm \text {,tot }}$ are not separately inviscid invariants of the axisymmetric Navier-Stokes equations, they do not a priori satisfy relations identical to Eq. (18). One can nevertheless consider "fluxlike" quantities [4]:

$$
\begin{aligned}
& \Pi_{E^{ \pm}}(k, t)=-\int_{0}^{k} T_{E^{ \pm}}\left(k^{\prime}, t\right) d k^{\prime}, \\
& \Pi_{H_{1}^{ \pm}}(k, t)=-\int_{0}^{k} T_{H_{1}^{ \pm}}\left(k^{\prime}, t\right) d k^{\prime},
\end{aligned}
$$

representing the flow of positively or negatively polarized energy and helicity out of the wave numbers lower than $k$. Unlike those associated with $E$ and $H_{1}$, these quantities are not expected to vanish as $k \rightarrow \infty$. The time averages of $\Pi_{E^{+}}$and $\Pi_{H_{1}^{+}}$[calculated over the time interval $t / \tau=[16,26]$ corresponding to the statistically steady state; see Fig. 5(a)] are plotted in Fig. 7. Like in the helical flow, the negative flow of (polarized) energy out of the wave numbers lower than large $k$ is found to be more important than the positive flow of (polarized) energy out of the wave numbers lower than small $k$, while the opposite occurs for polarized helicity. Even though the net helicity is zero, the polarized helicity is therefore preferably "transferred" towards the small scales and the polarized energy towards the large ones. However, the results obtained for the nonhelical flow cannot be straightforwardly interpreted as signatures of cascades of polarized energy and helicity since these quantities are not inviscid invariants of the dynamics (only the sums $E^{+}+E^{-}$and $H^{+}+H^{-}$are). This means that $E^{+}, E^{-}, H^{+}$, and $H^{-}$are not flux-conserving, but that a certain amount of each of them "leaks" to its oppositely polarized counterpart. The nonvanishing flux at large $k$ in Fig. 7(b) is associated to this effect. Indeed, in the case of the transfer of invariant quantities, the integral of the transfer over all wave numbers should vanish, so that the fluxes $\Pi_{E^{+}}$and $\Pi_{H_{1}^{+}}$should go to zero for $k$ tending to infinity. Incidentally, the flux of total energy in the nonhelical case (not shown here) behaves identically to that in the helical case [Fig. 4(a)] and to the flux of polarized energy in the nonhelical one [Fig. 7(a)]. In particular, an inverse energy cascade therefore occurs even in the absence of net helicity in the flow.

It is at this point interesting to discuss recent results on isotropic 3D turbulence, where also the fluxes in helical and nonhelical flows were considered [38]. The focus in that investigation was on the different types of triad interactions, in the Craya-Herring decomposition on helical modes. It was in particular observed that the different types of interactions and fluxes were only weakly influenced by the presence, or absence, of mean helicity. In particular, even in the absence of net helicity, the individual components of the helicity fluxes, considering the different possible triadic combinations 
of positively and negatively polarized modes, are large compared to the properly normalized energy fluxes. However, as in the present study, these fluxes sum to zero. This thereby illustrates how local helicity can play an important role in the nonlinear dynamics of turbulence, even when the net helicity is zero. A further decomposition of the different fluxes into homo- and heterochiral contributions, similar to that performed in Ref. [38], could be performed in the axisymmetric system.

\section{CONCLUSION}

The results presented in the present manuscript show that axisymmetric turbulence is indeed conceptually an intermediate system between 2D and 3D turbulence. As in three dimensions, the main invariants are the kinetic energy and the helicity, but unlike in three dimensions and as in two dimensions, the energy cascades towards the large scales as part of a dual cascade. Helicity is rather transferred towards the small scales. Even in the case in which the global helicity is zero, the same mechanism seems to persist. However, in that case the quantities transferred towards the small scales are the polarized helicity components. These are not strict invariants, but their fluxes, defined by analogy with the fluxes of conserved quantities, indicate a clear cascade-like mechanism.

These results explain why in Ref. [10] energy seems to be a more robust invariant than helicity, which is more rapidly dissipated under the effect of viscosity. The energy cascade towards the large scales also explains the tendency of axisymmetric turbulence to generate large-scale structures [10]. The presence of such coherent and persistent large-scale structures is a justification for attempts to understand the system using tools borrowed from statistical mechanics, as was done in Refs. [14-17].

The present work is further timely since a number of works have been recently dedicated to the investigation of the dynamics of 3D turbulence in which only a part of the helical modes is retained [35,36], or in which the weight of the different helical contributions is artificially modified [39]. It was shown that in such a triadically modified flow, the energy cascades towards the large scales, and helicity towards the small ones. In axisymmetric turbulence, this seems to be the case without numerical surgery, even though one could argue that the surgery has already taken place when removing the azimuthal variations from the dynamics. Taking this point of view, even 2D turbulence can be considered as an artificial modification of 3D turbulence.

A natural extension of the present work would consist in considering higher values of the Reynolds number, which would be made possible by the use of pseudospectral methods. The inertial ranges and associated scalings of the energy and helicity spectra could then be characterized more finely, in particular in the large wave number range.

\section{ACKNOWLEDGMENTS}

Shuojun $\mathrm{Li}$ is gratefully acknowledged for providing his code of direct numerical simulation of 2D turbulence [22]. The authors would like to thank Bérengère Dubrulle for suggesting that they carry out this investigation, and Kai Schneider for insightful comments on this work. B.Q.'s Ph.D. thesis was funded by a grant of the China Scholarship Council. This research was performed using the HPC resources provided by PMCS2I (École Centrale de Lyon).

[1] L. F. Richardson, Weather Prediction by Numerical Process (Cambridge University, Cambridge, 1922).

[2] A. N. Kolmogorov, The local structure of turbulence in incompressible viscous fluids at very large Reynolds numbers, Dokl. Akad. Nauk. SSSR 30, 299 (1941). Reprinted in Proc. R. Soc. London A 434, 9 (1991).

[3] A. Brissaud, U. Frisch, J. Leorat, M. Lesieur, and A. Mazure, Helicity cascades in fully developed isotropic turbulence, Phys. Fluids 16, 1366 (1973).

[4] Q. Chen, S. Chen, and G. L. Eyink, The joint cascade of energy and helicity in three-dimensional turbulence, Phys. Fluids 15, 361 (2003). 
[5] V. Borue and S. A. Orszag, Spectra in helical three-dimensional homogeneous isotropic turbulence, Phys. Rev. E 55, 7005 (1997).

[6] R. H. Kraichnan, Inertial ranges in two-dimensional turbulence, Phys. Fluids 10, 1417 (1967).

[7] R. H. Kraichnan, Inertial-range transfer in two-and three-dimensional turbulence, J. Fluid Mech. 47, 525 (1971).

[8] G. K. Batchelor, Computation of the energy spectrum in homogeneous two-dimensional turbulence, Phys. Fluids 12, II-233 (1969).

[9] G. Boffetta, Energy and enstrophy fluxes in the double cascade of two-dimensional turbulence, J. Fluid Mech. 589, 253 (2007).

[10] B. Qu, W. J. T. Bos, and A. Naso, Direct numerical simulation of axisymmetric turbulence, Phys. Rev. Fluids 2, 094608 (2017).

[11] E. Herbert, F. Daviaud, B. Dubrulle, S. Nazarenko, and A. Naso, Dual non-Kolmogorov cascades in a von Kármán flow, Europhys. Lett. 100, 44003 (2012).

[12] J. P. Laval, B. Dubrulle, and S. Nazarenko, Nonlocality and intermittency in three-dimensional turbulence, Phys. Fluids 13, 1995 (2001).

[13] J. Wesson and D. J. Campbell, Tokamaks (Oxford University Press, Oxford, 2011).

[14] N. Leprovost, B. Dubrulle, and P.-H. Chavanis, Dynamics and thermodynamics of axisymmetric flow: Theory, Phys. Rev. E 73, 046308 (2006).

[15] A. Naso, R. Monchaux, P.-H. Chavanis, and B. Dubrulle, Statistical mechanics of Beltrami flows in axisymmetric geometry: Theory reexamined, Phys. Rev. E 81, 066318 (2010).

[16] A. Naso, S. Thalabard, G. Collette, P.-H. Chavanis, and B. Dubrulle, Statistical mechanics of Beltrami flows in axisymmetric geometry: Equilibria and bifurcations, J. Stat. Mech. Theor. Exp. (2010) P06019.

[17] S. Thalabard, B. Dubrulle, and F. Bouchet, Statistical mechanics of the 3D axisymmetric Euler equations in a Taylor-Couette geometry, J. Stat. Mech. Theor. Exp. (2014) P01005.

[18] F. Ravelet, L. Marié, A. Chiffaudel, and F. Daviaud, Multistability and Memory Effect in a Highly Turbulent Flow: Experimental Evidence for a Global Bifurcation, Phys. Rev. Lett. 93, 164501 (2004).

[19] R. Monchaux, F. Ravelet, B. Dubrulle, A. Chiffaudel, and F. Daviaud, Properties of Steady States in Turbulent Axisymmetric Flows, Phys. Rev. Lett. 96, 124502 (2006).

[20] S. Thalabard, B. Saint-Michel, E. Herbert, F. Daviaud, and B. Dubrulle, A statistical mechanics framework for the large-scale structure of turbulent von Kármán flows, New J. Phys. 17, 063006 (2015).

[21] D. Montgomery and L. Turner, Two-and-a-half-dimensional magnetohydrodynamic turbulence, Phys. Fluids 25, 345 (1982).

[22] S. Li and D. Montgomery, Decaying two-dimensional turbulence with rigid walls, Phys. Lett. A 218, 281 (1996).

[23] S. Chandrasekhar and P. C. Kendall, On force-free magnetic fields, Astrophys. J. 126, 457 (1957).

[24] J. Boisson and B. Dubrulle, Three-dimensional magnetic field reconstruction in the VKS experiment through Galerkin transforms, New J. Phys. 13, 023037 (2011).

[25] Y. Kaneda, T. Ishihara, M. Yokokawa, K. Itakura, and A. Uno, Energy dissipation rate and energy spectrum in high resolution direct numerical simulations of turbulence in a periodic box, Phys. Fluids 15, L21 (2003).

[26] B. Qu, Numerical simulation of axisymmetric turbulence, Ph.D. thesis, École Centrale de Lyon, Université de Lyon, 2017.

[27] G. J. F. Van Heijst, H. J. H. Clercx, and D. Molenaar, The effects of solid boundaries on confined twodimensional turbulence, J. Fluid Mech. 554, 411 (2006).

[28] L. Biferale, M. Buzzicotti, and M. Linkmann, From two-dimensional to three-dimensional turbulence through two-dimensional three-component flows, Phys. Fluids 29, 111101 (2017).

[29] T. D. Lee, On some statistical properties of hydrodynamical and magneto-hydrodynamical fields, Q. Appl. Math. 10, 69 (1952).

[30] R. H. Kraichnan, Helical turbulence and absolute equilibrium, J. Fluid Mech. 59, 745 (1973).

[31] F. Waleffe, The nature of triad interactions in homogeneous turbulence, Phys. Fluids A 4, 350 (1992).

[32] L. Biferale, D. Pierotti, and F. Toschi, Helicity transfer in turbulent models, Phys. Rev. E 57, R2515 (1998).

[33] P. D. Ditlevsen and P. Giuliani, Anomalous scaling in a shell model of helical turbulence, Physica A (Amsterdam) 280, 69 (2000). 
[34] P. D. Ditlevsen and P. Giuliani, Dissipation in helical turbulence, Phys. Fluids 13, 3508 (2001).

[35] L. Biferale, S. Musacchio, and F. Toschi, Inverse Energy Cascade in Three-Dimensional Isotropic Turbulence, Phys. Rev. Lett. 108, 164501 (2012).

[36] L. Biferale, S. Musacchio, and F. Toschi, Split energy-helicity cascades in three-dimensional homogeneous and isotropic turbulence, J. Fluid Mech. 730, 309 (2013).

[37] P. D. Ditlevsen and P. Giuliani, Cascades in helical turbulence, Phys. Rev. E 63, 036304 (2001).

[38] A. Alexakis, Helically decomposed turbulence, J. Fluid Mech. 812, 752 (2017).

[39] G. Sahoo, A. Alexakis, and L. Biferale, Discontinuous Transition from Direct to Inverse Cascade in Three-Dimensional Turbulence, Phys. Rev. Lett. 118, 164501 (2017). 